\section{PO-0265 NON INVASIVE VENTILATION FOR SEVERE BRONCHIOLITIS}

S Mahdoui, A Borgi, N Ghali, A Hamdi, K Menif, A Bouziri, N Ben Jeballah. Intensive Care Unit, Children's Hospital, Tunis, Tunisia

\subsection{6/archdischild-2014-307384.919}

Introduction Non-invasive ventilation (NIV) is a relatively new ventilatory mode that has been increasingly used in the acute setting over the past 15 years, demonstrating beneficial effects in the paediatric population with different types of respiratory failure.

Objectives To examine whether infants with severe bronchiolitis could be managed with non-invasive ventilation (NIV) alone. To study the characteristics, clinical course and outcome of NIV patients.

Patients and methods A retrospective analysis was made of infants with severe bronchiolitis in a Paediatric Intensive Care Unit admitted from $01 / 09 / 2011$ to $31 / 01 / 2012$ and from $01 / 09 /$ 2012 to $31 / 02 / 2013$. One thousand and sixty-four infants with severe bronchiolitis were admitted. One thousand and two were invasively ventilated, seventy-two were treated with NIV. We aimed to examine the characteristics, clinical course and outcome for those who received NIV.

Results Seventy-two patients, including 6 with apnea, were treated exclusively with NIV. The mean age was de 54,2 days \pm 39,1 (8-221). The mean respiratory rate was 61 breaths/min $\pm 16,7$ (20-104). NIV was delivered by continuous (CPAP) in seven patients, bi-level (BiPAP) positive airway pressure in thirtyfour infants and high-flow nasal cannula in thirty-one patients. Twenty-three failed to respond and were invasively ventilated. Risk factors for NIV failure were prematurity and bacterial infection. Duration of hospital stay was shorter in responders. There were no major complications related with NIV.

Conclusion This study demonstrates the efficacy of NIV as a form of respiratory support for infants with severe bronchiolitis avoiding ETI in most of the patients. Risk factors for failure were related with immaturity and severe infection.

\section{P0-0266 SEPTIC SHOCK SECONDARY TO A COMMUNITY ACQUIRED INFECTION: ABOUT 51 CASES}

S Mahdoui, S Tilouche, A Tej, N Soyah, N Missaoui, N Kahloul, A Mlika, J Bouguila, L Boughamoura. 'Pediatrics, Farhat Hached Hospital, Sousse, Tunisia

\subsection{6/archdischild-2014-307384.920}

Introduction Septic shock in children remains one of the main causes of morbidity and mortality worldwide. Although their diagnosis and their management is largely influenced by studies done in adults. There are important considerations relevant for paediatrics.

Goal This study had for aim to evaluate epidemiology and outcome of septic shock secondary to a community acquired infection.

Patients and methods A retrospective analysis was made of patients admitted between January 2004 and December 2013, in a paediatric department for septic shock secondary to a community-acquired infection. Neonates were excluded from the study.

Results Fifty-one cases were included. The average age was 2.7 years (1 month-14 years). The average time between the observation of first disease symptoms and admission was 2.8 days (114 days). The average PRISM during the first 24 h was 20.3 (4-
41). Multiple organ failure was present in the majority of cases (96\%). Gram-negative bacteria were the predominant pathogens $(50 \%)$. Respiratory infection is the most common infection site (37.3\%). The empiric therapy was a combination of Cefotaxime and Aminoglycoside in 52.9\% of cases. Dopamine remains the most prescribed catecholamine $(72.5 \%)$. Dobutamine and Norepinephrine were used in $62.7 \%$ and $31.4 \%$ of cases. Mechanical ventilation was needed in 39 patients with an average of 2.8 days (1-16 days). The average length of hospitalisation was 12.6 \pm 6.9 days (4-30 days). The mortality was $70.6 \%$.

Conclusion Despite significant progress in the understanding and treatment, septic shock continues to be a major health problem in developing countries and around the world.

\section{PO-0267 SECONDARY TRANSPORT OF CRITICAL PAEDIATRIC PATIENTS: SURVEY IN LOMBARDY}

'S Maiandi, ${ }^{2} \mathrm{M}$ Tinelli, ${ }^{3} \mathrm{~B}$ Cantoni. ${ }^{1}$ Pediatric Unit and Pediatric Emergency Unit, Azienda Ospedaliera della Provincia di Lodi, Lodi, Italy; ${ }^{2}$ Università degli Studi di Milano, Corso Di Laurea in Infermieristica Pediatrica, Milano, Italy; ${ }^{3}$ SITRA Area Delle Pediatrie, Fond. IRCCS Cà Granda Ospedale Maggiore Policlinico, Milano, Italy

\subsection{6/archdischild-2014-307384.921}

Background The current health network structure and a lack of a proper filter for pre-hospital care makes every hospital potentially involved into the management of any critical paediatric patient regardless the local experience and organisation.

Any first patient's stabilisation will have to be followed by a secured secondary transport until the hospitalisation at the new facility.

Objective Explore the hospitals' organisational set up concerning the secondary transport of paediatric critical care patients.

Materials and methods Structured survey delivered to 92 hospitals in Lombardy

Results The response rate was 56\%, corresponding to 52 health care facilities. In 29 facilities a dedicated transport service for critical care patients does exist but just in one hospital it's specific for paediatric patients and it has dedicated staff. Forty facilities are equipped with a paediatric medical bag and the more involved operator is an anesthesiologist for 39\% of cases, followed by the paediatrician in $13 \%$ of cases. The nurse participates to the transport in $50 \%$ of cases; in $28 \%$ of the hospitals a critical care nurses is involved, in $7 \%$ of cases the nurse is not specialised and in $4 \%$ the nurses is specialised in paediatrics.

Discussion The data shows an non homogeneity management of the critical care patient secondary transport.

Conclusions It's highly desirable the activation of a secondary transport service with an organisational level compared to the neonatal emergency transport service because the child has its own characteristics as like as the newborn or the adult.

\section{PO-0268 THE PARENTAL PRESENCE DURING PAEDIATRIC CARDIOPULMONARY RESUSCITATION: EPIDEMIOLOGICAL ANALYSIS}

${ }^{1} \mathrm{~S}$ Maiandi, ${ }^{2} \mathrm{~S}$ Mondini, ${ }^{3} \mathrm{~B}$ Cantoni. ${ }^{1}$ Pediatric Unit and Pediatric Emergency Unit, Azienda Ospedaliera della Provincia di Lodi, Lodi, Italy; ${ }^{2}$ Università Degli Studi Di Milano, Corso Di Laurea in Infermieristica Pediatrica, Milano, Italy; ${ }^{3}$ SITRA Area Delle Pediatrie, Fond. IRCCS Cà Granda Ospedale Maggiore Policlinico, Milano, Italy

10.1136/archdischild-2014-307384.922

Background The paediatric cardiopulmonary resuscitation involves high level skills by operators in a setting characterised 
by an high complexity and a considerable emotional impact. Clinical experience shows that parents are often turned away from the emergency room because they were considered an obstacle.

Objective Investigate the behaviour of some hospitals about the management of parents during cardiopulmonary resuscitation in paediatric subjects.

Materials and Methods Deliver a questionnaire to the nurse coordinators of 19 Italian hospital.

Results The questionnaire had a response rate equal to $89.4 \%$, corresponding to 17 hospitals.

$23.5 \%$ of hospitals admit the presence of both parents during all phases of resuscitation without age limits, while $17,7 \%$ of the structures do not allow the parental presence because of the possible fear created by the anxiety of parents, for the inadequacy of the spaces within the operational units and the perception that parents can potentially be an obstacle for health professionals during the resuscitation procedures.

The remaining 58.8\% have a favourable opinion about the possibility of guaranteeing to the paediatric patient, without any age limit, the presence of parents during all phases of the cardiopulmonary resuscitation but difficulties arise to make such a guarantee for the inadequacy of the spaces, for difficulties in managing the behaviour of the relatives especially in regards of anxiety and for the absence of any psychological support figure. Conclusions It's necessary that all the hospitals in any way involved into the primary care process shall act in conformity with common management protocols concerning this theme.

\section{P0-0269 CHARACTERISTICS OF CLINICAL PATHWAYS AND EMERGENCY CARE IN PAEDIATRIC SURGICAL PATHOLOGY IN LOMBARDY}

${ }^{1} \mathrm{~S}$ Maiandi, ${ }^{2} \mathrm{E}$ Gabos, ${ }^{3} \mathrm{~B}$ Cantoni. ${ }^{1}$ Pediatric Unit and Pediatric Emergency Unit, Azienda Ospedaliera Della Provincia Di Lodi, Lodi, Italy; ${ }^{2}$ Università Degli Studi Di Milano, Corso Di Laurea in Infermieristica Pediatrica, Milano, Italy; ${ }^{3}$ SITRA Area Delle Pediatrie, Fond. IRCCS Cà Granda Ospedale Maggiore Policlinico, Milano, Italy

\subsection{6/archdischild-2014-307384.923}

Background The clinical and emergency care daily activities show that only a fraction of children with urgent surgical pathology access to dedicated paediatric centres while most of the diagnostic, therapeutic and emergency care takes place at facilities not necessarily specialised in the treatment of paediatric acute illness; as it should be as per regional and national legislation.

Objective Analyse the care pathways of children hospitalised for urgent surgical pathology (appendicitis, hypertrophic pyloric stenosis).

Materials and methods Structured survey delivered to 93 hospitals in Lombardy.

Results The response rate was equal to $79.7 \%$, corresponding to 74 facilities: the appendectomy was performed in $90.5 \%$ ( $\mathrm{n}=$ 67 ) and the surgical correction of HPS was performed in $17.6 \%$ $(\mathrm{n}=13)$ of cases.

The hospitalisation was in $14.9 \%$ of cases $(n=10)$ at the paediatric surgery department, $56.7 \%(\mathrm{n}=38)$ at paediatrics department, $4.5 \%(\mathrm{n}=3)$ at general surgery department having rooms dedicated to children and $23.9 \%(n=16)$ at general surgery department without paediatrics dedicated rooms.

Discussion The analysis shows that the regional and national legislation is disregarded: it is alarming the attitude of 19 hospitals that hospitalise children in unsuitable environments, with rooms shared with adults and aged patients and the correlated risk of adopting care pathways "not dedicated and unsafe " for children.

Conclusions It's desirable an adjustment of the hospital facilities to promotes the creation of a culture in paediatrics able to ensure the highest levels of care in such a vulnerable age.

\section{PO-0270 USE OF SUCROSE AND NON-PHARMACOLOGICAL METHODS IN ALLEVIATING NEWBORN PROCEDURAL PAIN IN A PICU SETTING}

A Manou. Neonatal Medicine, Leeds Teaching Hospitals, Leeds, UK

\subsection{6/archdischild-2014-307384.924}

Background Sucrose has become standard practice across neonatal units for reducing procedural pain in preterm and term newborns. In infants admitted to PICU subject to similar procedures, systemic analgesics like morphine remains the preferred choice for procedural pain despite emerging links between these agents and adverse neurodevelopment outcomes.

Aim To explore the use of Sucrose and other non-pharmacologic methods for alleviating neonatal procedural pain in a PICU setting.

Methods Online survey sent to all medical and nursing staff in a regional PICU about use of sucrose and non-pharmacological methods for procedural pain relief in newborns.

Results There were a total of 28 respondents.

PICU staff are aware of Sucrose and non-pharmacological methods. However, they do not use these routinely for procedural pain, and $18 \%$ would never use a non-pharmacological method or sucrose.

$18 \%$ staff members do not believe these methods to be effective in controlling pain in newborns.

The reasons for not using these measures routinely were that these are unfeasible in post-operative patients (50\% respondents) and not required in patients already on morphine (7\% respondents). $25 \%$ respondents said other staff members discouraged them if they wanted to use these measures. $43 \%$ respondents want Sucrose as a part of the guideline for pain management in babies.

Conclusions Despite awareness of sucrose and non-pharmacological adjuncts, these are not consistently used in PICU. Incorporating these methods as a part of evidence based guideline for management of procedural pain can reduce the need for systemic analgesia and reduce the adverse neurodevelopmental side effects.

\section{P0-0271 A DIFFICULT DIAGNOSIS OF NATURAL KILLER CELL LYMPHOMA PRESENTING WITH BILATERAL PLEURAL EFFUSIONS}

${ }^{1}$ A Meral, ${ }^{2} S$ Kesici, ${ }^{2} \mathrm{M}$ Tanyildiz, ${ }^{3} \mathrm{Z}$ Akcoren, ${ }^{4} \mathrm{~B}$ Aydin, ${ }^{1} \mathrm{~A}$ Birbilen, ${ }^{2} \mathrm{~B}$ Bayrakci. ${ }^{1}$ Department of Pediatrics, Hacettepe University Faculty of Medicine, Ankara, Turkey; ${ }^{2}$ Department of Pediatrics Division of Pediatric Intensive Care, Hacettepe University Faculty of Medicine, Ankara, Turkey; ${ }^{3}$ Department of Pediatrics Division of Pediatric Pathology, Hacettepe University Faculty of Medicine, Ankara, Turkey; ${ }^{4}$ Department of Pediatrics Division of Pediatric Oncology, Hacettepe University Faculty of Medicine, Ankara, Turkey

\subsection{6/archdischild-2014-307384.925}

Background Natural killer (NK) cell lymphoma is a rare and aggressive neoplasm characterised by angioinvasion or angiodestruction. The aim of this report to describe a patient with an 